\title{
Modeling Pulsed-Laser Melting of Embedded Semiconductor Nanoparticles
}

\author{
C. A. Sawyer ${ }^{1,2}$, J. Guzman ${ }^{1,2}$, C. N. Boswell-Koller ${ }^{1,2}$, \\ M. P. Sherburne ${ }^{1,2}$, J. P. Mastandrea ${ }^{1,2}$, K. C. Bustillo ${ }^{1,2}$, \\ J. W. Ager III' ${ }^{2}$, E. E. Haller ${ }^{1,2}$, D. C. Chrzan ${ }^{1,2}$ \\ ${ }^{1}$ Department of Materials Science and Engineering, University of \\ California, Berkeley, CA 94720, USA \\ 2 Materials Sciences Division, Lawrence Berkeley National Laboratory, \\ Berkeley, CA 94720, USA
}

\begin{abstract}
Pulsed-laser melting (PLM) is commonly used to achieve a fast quench rate in both thin films and nanoparticles. A model for the size evolution during PLM of nanoparticles confined in a transparent matrix, such as those created by ion-beam synthesis, is presented. A self-consistent mean-field rate equations approach that has been used successfully to model ion beam synthesis of germanium nanoparticles in silica is extended to include the PLM process. The PLM model includes classical optical absorption, multiscale heat transport by both analytical and finite difference methods, and melting kinetics for confined nanoparticles. The treatment of nucleation and coarsening behavior developed for the ion beam synthesis model is modified to allow for a non-uniform temperature gradient and for interacting liquid and solid particles with different properties. The model allows prediction of the particle size distribution after PLM under various laser fluences, starting from any particle size distribution including as-implanted or annealed simulated samples. A route for narrowing the size distribution of embedded nanoparticles is suggested, with simulated distribution widths as low as $15 \%$ of the average size. This work is supported by the US Department of Energy under Contract No. DE-AC02-05CH11231.
\end{abstract}

\section{Introduction}

Phase change materials are studied widely for high-density data storage applications; a current system of interest is semiconductor or semiconductor-metal alloy nanoparticles 
confined in a transparent matrix[1]. Phase change applications require a method of reliably switching the material from one phase to the other. One such method is thermal; a fast quench from pulsed-laser melting (PLM) will produce amorphous particles while a slower rapid thermal anneal (RTA) will cause recrystallization[1, 2, 3]. In PLM, a short laser pulse produces rapid temperature rise in the sample, causing melting, dissolution, and/or coarsening of the nanoparticles; a quantitative understanding of this process will enable control and optimization of future devices.

For example, an ongoing problem in nanoscience is controlling the width of the size distribution of nanoparticles to better measure and take advantage of size-dependent properties. Wet chemistry methods have reached size distributions with relative width as low as 9\%[4] via "size focusing." A similar narrowing of nanoparticles grown by other means, including embedded particles made by ion-beam synthesis, might be achieved by suitable heat treatments including PLM.

A quantitative model of the synthesis of semiconductor nanoparticles has been created and shown to agree with experiment $[5,6]$, but PLM requires a more detailed model. The laser absorption, heat transport, phase, size, and size distribution of the particles are all interrelated. In particular, the melting transition and the properties of the melt have a strong impact on the results of the process. Because PLM involves a short (e.g. $30 \mathrm{~ns}$ ) laser pulse and nanoscale particles, observing the process in situ is experimentally difficult. Thus, a detailed numerical simulation is desired.

Previous models of the PLM process $(e g[7,8])$ have focused on thin films of semiconductor or metal, in which a surface layer absorbs the laser homogeneously, and heat transport and melting are essentially one-dimensional. Dewetting and pattern formation in very thin films under PLM $[9,10]$ have also been observed and modeled. The temperature evolution, melt front location, and diffusion of dopant elements are often tracked in these models; in the current model the melt front is replaced by the numbers of solid and liquid clusters, and diffusion is complicated by the interaction of clusters and a matrix with low solubility.

\section{Methods}

A self-consistent mean-field rate equation model following $[5,6,11]$ and $[12,13]$ is developed. The model includes implantation of ions, nucleation and growth of clusters, ion impingement-induced fragmentation of clusters, and melting and freezing transitions. The rate equations track a set of spatially averaged concentrations of clusters of each possible size and phase. The effect of the laser is treated by a simple optical absorption model and the temperature in the sample is tracked by a combination of analytical and finite difference solutions of the heat equation.

The key physical phenomena which make PLM of embedded nanoparticles an interesting problem to model are related to melting: first, melting is complicated by the relative importance of interface energies in nanoparticles. A significant hysteresis in 
the melting/freezing point has been observed in these systems, and is explained by a relatively simple kinetic model[14]. This melting point shift is size-dependent, so for a significant amount of time a population of liquid particles is interacting with solid particles. This complicates the coarsening problem, as liquid particles of the same size will have a different chemical potential than solid particles. These two related phenomena serve to differentiate nanoparticle PLM from an ordinary macroscale coarsening problem. Additionally, the small sizes of the particles allow atom-by-atom modeling of the growth and coarsening process.

The system modeled is germanium implanted into a 500-nm thick layer of silica on a thick silicon substrate. An implanted layer forms at the mean implant depth of the ions with a thickness of approximately $100 \mathrm{~nm}$, starting $100 \mathrm{~nm}$ below the silica surface. The volumetric dose in this layer is $.004 \AA^{-3}$, corresponding to a $4 \times 10^{16} \mathrm{~cm}^{-2}$ experimental dose. It should be noted that by assumption the model is only valid for relatively low volume fractions of implanted material, for which the particles can be considered isolated spheres.

The laser absorption and heat transport models will be discussed first, assuming a distribution of particles $\left\{n_{s}\right\}$, where $s$ denotes the number of atoms in a cluster and $n_{s}$ the number per unit volume of clusters of that size. For a discussion of the ionimplantation portion of the model, which creates the starting distributions used here, see $[5,6,11]$. The evolution of this particle distribution will follow in sections 2.3-2.4, and the treatment of molten particles will be discussed last.

\subsection{Light Absorption}

To model the behavior during PLM, the fundamental mechanism is assumed to be absorption of light by the nanoparticles, which causes a large temperature rise in the sample. The silica matrix is transparent to the wavelength used for PLM, so absorption by the silica is neglected. It is assumed that absorption happens instantaneously relative to the time scale of the model, i.e. that the duration of electronic excitation and nonradiative relaxation which take place on the scale of picoseconds can be neglected. To estimate the absorption, the particles are treated as a film with equivalent volume to the total particle volume. This reduces to a one-dimensional optics problem. The laser beam intensity is assumed to be reduced by reflection at the air/silica and silica/germanium interfaces, and reflection from the back surface of the Ge layer and from the Si substrate are expected. Including all internal reflections within the Ge layer and between the Ge and substrate surfaces, the total absorbed intensity is

$$
\begin{gathered}
I_{a b s}=I_{0}\left(1-R_{a}\right)\left(1-R_{G}\right)\left(1-\exp \left(-\alpha_{G e} h\right)+\exp \left(-\alpha_{G e} h\right)\left(R_{G} I_{1}+\left(1-R_{G}\right) I_{2}\right)\right) \\
I_{1}=1-\exp \left(-\alpha_{G e} h\right)+\exp \left(-\alpha_{G e} h\right) R_{1}\left(R_{G} I_{1}+\left(1-R_{G}\right) I_{2}\right) \\
I_{2}=R_{S}\left(\left(1-R_{G}\right) I_{1}+R_{G} I_{2}\right) .
\end{gathered}
$$


Here $R_{a}, R_{G}$ and $R_{S}$ are the reflectances at the air- $\mathrm{SiO}_{2}, \mathrm{Ge}-\mathrm{SiO}_{2}$ and $\mathrm{Si}-\mathrm{SiO}_{2}$ interfaces respectively; $\alpha_{G e}$ is the absorption coefficient of the germanium, $h$ is the equivalent layer thickness, and $I_{0}$ is the incident intensity of the laser. The functions $I_{1}$ and $I_{2}$ describe a beam of light bouncing within the Ge layer and within the silica layer between particles and substrate, respectively. The total absorbed intensity thus calculated is then divided by the equivalent layer thickness to get an average volumetric power density in effect within each nanoparticle.

It should be noted that the equivalent layer approximation provides an upper bound for the energy absorbed. In reality the particles are non-flat and are randomly placed throughout the sample; reflections will be random and a full scattering solution would be needed. Instead, a lower bound is postulated: the laser is taken to pass once through the equivalent layer and then is dispersed. The intensity absorbed is then simply

$$
I_{a b s}=I_{0}\left(1-R_{a}\right)\left(1-R_{G}\right)\left(1-\exp \left(-\alpha_{G e} h\right)\right) .
$$

The different results of these two bounds will be discussed in section 3.1; fitting to future experiments would determine which bound is more appropriate.

A small fraction of the laser energy is transmitted into the silicon substrate; this energy is taken to be absorbed according to $P(x)=I_{S i} \alpha \exp \left(-\alpha_{S i} x\right)$ and be a small source of heat. The magnitude of $I_{S i}$, the intensity entering the silicon substrate, is calculated from the upper bound as

$$
I_{S i}=\frac{I_{0} \exp \left(-\alpha_{G e} h\right)\left(1-R_{a}\right)\left(1-R_{G}\right)^{2}\left(1-R_{S}\right)}{1-R_{G} R_{S}-\exp \left(-\alpha_{G e} h\right)^{2} R_{G}\left(R_{S}+R_{G}\left(1-2 R_{S}\right)\right)} .
$$

The optical properties of the germanium are temperature-dependent, especially at the melting transition. The dielectric function changes continuously with temperature in solid germanium[15]. At the melting transition, the reflectivity jumps discontinuously from $55 \%$ to $65 \%[16]$, while the absorption coefficient is high enough for both hot solid and liquid to defy measurement of the difference.

\subsection{Temperature Evolution}

Heat generated in the clusters absorbing laser light is conducted rapidly out of the nanoclusters into the surrounding matrix. The implanted layer itself is flanked by layers of unimplanted silica and then a silicon substrate on one side and air on the other. A two-tiered model is used for the temperature evolution; first the heat transport out of each nanoparticle is calculated on the angstrom scale and then the transport in the various layers is calculated on a hundred-nanometer scale. The first tier is an analytical solution to the heat equation in spherical symmetry with an effectively infinite matrix; the second is a finite difference model of conduction and radiation in one dimension normal to the sample surface. 
An analytical solution of the heat equation in spherical symmetry is used following[17]. The equation is solved within a spherical region of one material under a constant volumetric power density surrounded by an infinite matrix of a second material with zero power density. The temperature and heat flux are constrained to be continuous across the interface, neglecting any contact resistance; the temperature is constrained to be finite at $r=0$ and to go to a base temperature $T_{0}$ at $t=0$ or $r \rightarrow \infty$. The temperature rise $\Delta T=T-T_{0}$ is given by

$$
\Delta T(r \leq R, t)=\frac{P R^{2}}{3 \lambda_{2}}\left[1+\frac{q_{\lambda}}{2}\left(1-\frac{r^{2}}{R^{2}}\right)+\frac{6}{\pi} q_{\lambda}^{3 / 2} q^{1 / 2} \frac{R}{r} \int_{0}^{\infty} f(z, r, t) g(z, r) d z\right]
$$

with $R$ the radius of the particle, $P$ the volumetric power input, and the abbreviations

$$
\begin{gathered}
q_{\lambda}=\frac{\lambda_{2}}{\lambda_{1}} \quad q=\frac{\rho_{2} c_{2}}{\rho_{1} c_{1}} \quad g(z, r)=\sin \frac{r z}{R} \\
f(z, r, t)=z^{-2} \exp \left(-\frac{\lambda_{1} t z^{2}}{\rho_{1} c_{1} R^{2}}\right)\left(\frac{z \cos z-\sin z}{\left(\left(q_{\lambda}-1\right) \sin z+z \cos z\right)^{2}+q_{\lambda} q(z \sin z)^{2}}\right) .
\end{gathered}
$$

The $\lambda_{1}, \rho_{1}$, and $c_{1}$ are the thermal conductivity, density, and heat capacity of the particle while $\lambda_{2}, \rho_{2}$, and $c_{2}$ are those of the matrix. This solution is valid only within the particle $(r<R)$; the matching solution (see [17]) for $r>R$ is here omitted for brevity.

Of course, the particle is not isolated in an infinite matrix; the sample contains many particles close together. In the mean-field framework it is not possible to exactly calculate the heat transport among the randomly-located particles. Instead the matrix is taken to have a uniform temperature that is the spatial average of the true temperature profile. This temperature is calculated by totaling the heat transfer from all the particles during time step $\Delta t$ and using this as a uniform power to the implanted layer, both particles and matrix. The two power sources within the nanoparticles are taken to superpose, so the temperature at the particle edge is that calculated by the spherical solution plus that of the implanted layer. The combination of the infinite-matrix approximation with the uniform temperature approximation is contradictory, however heat conservation is ensured and a reasonable approximation for heat retention in the particles is obtained without extremely computationally-intensive angstrom-scale finite difference methods.

Because the implanted layer is not infinite in extent, there is a second heat transfer problem; the heat in the implanted layer diffuses into the adjacent unimplanted silica, the silicon substrate and the air. In addition, at higher temperatures radiative heat transfer from the silica layer into the air becomes significant. The multilayered sample geometry and the interplay of conductive and radiative heat transfer call for a finite difference approach. Finite difference heat conduction is computed according to

$$
\frac{\partial T}{\partial t}=\nabla \cdot(a \nabla T)+\Phi(x)
$$


with $a(x)=\frac{\lambda}{c \rho}$ the thermal properties of the material at location $x . \Phi(x)$ is any volumetric power modified by $\frac{1}{c \rho}$; within the implanted layer this is the power output from the nanoparticles,

$$
\Phi(x \in \text { implanted })=\frac{1}{c \rho}\left(P \sum_{s} n_{s} V_{s}-\sum_{s} n_{s} \int_{V_{s}} c \rho \frac{\Delta T}{\Delta t} d V\right) .
$$

The radiative heat loss from the silica surface is treated as another $\Phi$. For simplicity, the silica is approximated as a blackbody and radiative power is computed from the Stefan-Boltzmann law

$$
P_{\text {rad }}=\sigma A T^{4}
$$

with $\sigma$ the Stefan-Boltzmann constant and $A$ the area emitting. In the finite difference framework, the element at the silica surface is given a power term

$$
\Phi\left(x_{\text {surface }}\right)=\frac{\sigma T(x)^{4}}{c \rho \Delta x} .
$$

The finite difference simulation takes boundary conditions $T=T_{0}$ as $x \rightarrow \pm \infty$ that are implemented by creating large enough air and silicon layers that the temperature rise at the simulation edges is insignificant in the simulation time and setting $T(0)=$ $T\left(x_{\max }\right)=T_{0}$. Thermal resistance at all interfaces is neglected, giving the additional condition that the temperature is the same coming from either side of a boundary.

The finite difference simulation is run for multiple timesteps between each update of the particle size distribution; though the temperature profile and the size distribution should evolve in parallel this would be computationally costly for a very small gain in accuracy. Absorption and transport out of the nanoparticles are also calculated on the larger timestep, and the power to the matrix is averaged over this time. At each update of the particle distribution, the temperature of the particles is set to that of the matrix and the heat retained is added to the matrix power for the following timestep; because this heat will be rapidly conducted out of the particles this approximation is reasonable.

\subsection{Nucleation, Growth and Coarsening}

Ions implanted into a matrix in which their solubility is low tend to precipitate into clusters. These clusters act as sinks to which monomers diffusing through the matrix attach. The clusters also desorb monomers to keep an equilibrium between dissolved monomers and the cluster surface. The derivation of the rates of these two processes follows that of [5] and [12], but accounts for the internal temperature gradients that were not needed in previous work.

Identifying an average time between thermal desorption events from an $s$-cluster as $\tau_{s}$ and a capture number $\sigma_{s}$ such that $D \sigma_{s}\left\langle n_{1}\right\rangle$ gives the rate for an $s$-cluster to capture 
monomers, we construct rate equations for nucleation, growth and coarsening as

$$
\begin{aligned}
\frac{d\left\langle n_{1}\right\rangle}{d t} & =-2 D \sigma_{1}\left\langle n_{1}\right\rangle^{2}-D \sum_{j>1} \sigma_{j}\left\langle n_{j}\right\rangle\left\langle n_{1}\right\rangle+2 \frac{\left\langle n_{2}\right\rangle}{\tau_{2}}+\sum_{j>2} \frac{\left\langle n_{j}\right\rangle}{\tau_{j}} \\
\frac{d\left\langle n_{s}\right\rangle}{d t} & =D \sigma_{s-1}\left\langle n_{s-1}\right\rangle\left\langle n_{1}\right\rangle-D \sigma_{s}\left\langle n_{s}\right\rangle\left\langle n_{1}\right\rangle-\frac{\left\langle n_{s}\right\rangle}{\tau_{s}}+\frac{\left\langle n_{s+1}\right\rangle}{\tau_{s+1}} .
\end{aligned}
$$

The capture numbers $\sigma_{s}$ and inverse desorption rates $\tau_{s}$ are in general unknown, but can be calculated by a self-consistent mean-field method. Consider a spherical $s$-cluster in a monomer field $n_{1}(\vec{r}, t)$ and a set of mean fields of $j$-particles $\left\langle n_{j}\right\rangle$. The monomer field follows a diffusion-reaction equation,

$$
\frac{\partial n_{1}(\vec{r}, t)}{\partial t}=\nabla \cdot\left(D \nabla n_{1}(\vec{r}, t)\right)+\mathcal{J}-D \xi^{-2} n_{1}(\vec{r}, t)
$$

where the first term covers diffusion, the second the addition of monomers to the matrix by detachment from the set of $j$-particles, and the third depletion of monomers by attachment to $j$-particles, where $\xi$ is the average distance traveled by a monomer before capture. The associations

$$
\begin{gathered}
\mathcal{J}=2 \frac{\left\langle n_{2}\right\rangle}{\tau_{2}}+\sum_{j>2} \frac{\left\langle n_{j}\right\rangle}{\tau_{j}} \\
\xi^{-2}=2 \sigma_{1}\left\langle n_{1}\right\rangle+\sum_{j>1} \sigma_{j}\left\langle n_{j}\right\rangle
\end{gathered}
$$

are made in equation 13 , giving

$$
\frac{d\left\langle n_{1}\right\rangle}{d t}=-D \xi^{-2}\left\langle n_{1}\right\rangle+\mathcal{J}
$$

Combining equations (15) and (18) and making the definition $\eta \equiv n_{1}-\left\langle n_{1}\right\rangle$,

$$
\frac{\partial \eta}{\partial t}=\frac{\partial n_{1}}{\partial t}-\frac{d\left\langle n_{1}\right\rangle}{d t}=\nabla \cdot(D \nabla \eta)-D \xi^{-2} \eta \approx 0 .
$$

It is assumed here that $\frac{\partial n_{1}}{\partial t} \approx \frac{d\left\langle n_{1}\right\rangle}{d t}$ and $n_{1}(r) \approx\left\langle n_{1}\right\rangle$, i.e. that the concentration of monomers does not differ much from the average concentration.

Because the diffusion constant $D=D_{0} \exp \left(-E_{m} / k T\right)$ depends on the temperature, which varies spatially within the sample, the simplification $\nabla \cdot\left(D \nabla n_{1}\right)=D \nabla^{2} n_{1}$ may not be made as it is in [5] and [12]. The equation is not analytically solvable without this simplification; instead, a finite-difference solution is used. To make the problem tractable, the temperature is approximated as having a simple $r^{-1}$ dependence as in the steady state $T=T_{0}+\frac{P R^{3}}{3 \lambda_{2} r}$; the calculated particle edge temperature is used instead of $\frac{P R^{2}}{3 \lambda_{2}}$. The diffusion constant as a function of $r$ is then

$$
D=D_{0} \exp \left(\frac{-E_{m}}{k\left(T_{0}+\frac{R \Delta T}{r}\right)}\right) .
$$


The object of the finite difference calculation is to identify $D \sigma$ and $\tau$ from

$$
J_{s}=\frac{1}{\tau_{s+1}}-D \sigma_{s}\left\langle n_{1}\right\rangle
$$

with $J_{s}$ the flux out of an $s$-cluster consistent with equation (13); $D$ is measured at the particle surface. Two additional formulations of $J_{s}$ are used, an atomistic picture and the definition

$$
J_{s} \equiv-4 \pi\left(R_{s}^{\prime}\right)^{2}\left(D \frac{\partial n_{1}}{\partial r}\right)_{R_{s}^{\prime}} .
$$

The $R_{s}^{\prime} \equiv R_{s}+r_{c a p}$ is a radius defined by the physics of attachment; $R_{s}$ is the physical radius of the particle and $r_{c a p}$ is a small additional capture radius within which monomers can be assumed to already have crossed the energy barrier to attachment and within which the diffusion-reaction equation (15) does not hold[12]. This is the radius which monomers must cross to attach or detach from the particle, and so the flux at this location is identically that of equation (21). An atomistic picture of the flux is given by

$$
J_{s}=4 \pi R_{s+1}^{2} \frac{D}{a} n_{\infty} e^{\left(\Gamma / R_{s+1}\right)}-4 \pi\left(R_{s}^{\prime}\right)^{2} \frac{D}{a} n_{1}\left(R_{s}^{\prime}\right) .
$$

This equation arises from $4 \pi\left(R_{s}^{\prime}\right)^{2} n_{1}\left(R_{s}^{\prime}\right)$ total monomers populating this shell, $1 / 6$ of which will diffuse inward at a speed $6 D / a[5]$. These monomers are leaving the monomer field. At the same time the particle is thermally desorbing monomers to maintain an equilibrium with the matrix; the concentration of monomers at the particle surface is $n_{\infty} e^{\left(\Gamma / R_{s+1}\right)}$, with $n_{\infty}$ the solubility at a flat surface and $\Gamma$ the capillary length. Again, $1 / 6$ of these will diffuse outward at a speed $6 D / a$. Equations (23) and (21) may both be broken loosely into attachment and detachment terms; however, the division is not exact. Equation (23) considers the monomers leaving the $R_{s+1}$ radius without considering whether they reached that location by detachment from the particle or by diffusion through the matrix, for example. Instead, the identification is made by noting that equation (21) has a linear dependence on $\left\langle n_{1}\right\rangle$; terms with a dependence on $\left\langle n_{1}\right\rangle$ belong with $D \sigma$ while the rest belong with $\frac{1}{\tau}$. In the case that $D$ is constant, this identification may be made analytically $[5,12]$, but otherwise the identification is made by calculating the $\left\langle n_{1}\right\rangle$-derivative of the flux $J_{s}$. This is done in the finite-difference approximation by conducting the finite difference calculation of the local monomer density outside each particle twice using $\left\langle n_{1}\right\rangle \pm \delta\left\langle n_{1}\right\rangle$.

The finite difference model considers a spherically-symmetric region surrounding an $s$-particle from the radius $R_{s}^{\prime}$ to a large $R_{\infty}$. The boundary condition at $R_{s}^{\prime}$ is a Robin boundary condition, given by equating (22) and (23) and solving for $\frac{\partial \eta}{\partial r}$. The boundary condition at $R_{\infty}$ is $\eta=0$, which is the self-consistency condition

$$
\lim _{r \rightarrow \infty} n_{1}=\left\langle n_{1}\right\rangle .
$$


The initial condition is $\eta(r)=0$, and the integration time is sufficient to let the system come to the steady state, i.e. $\frac{\partial \eta}{\partial t}=0$.

The controlling equation (19) includes a dependence on $\xi$, which in turn depends on the set $\left\{\sigma_{s}\right\}$. This difficulty is removed by guessing a value of $\xi$, then calculating $\left\{\sigma_{s}\right\}$ and updating $\xi$ until the values of $\xi$ converge. The values of $\sigma$ and $\tau$ are found for each particle size as

$$
\begin{gathered}
\sigma_{s}=\frac{-1}{D\left(R_{s}^{\prime}\right)} \frac{\partial J_{s}}{\partial\left\langle n_{1}\right\rangle} \\
\frac{1}{\tau_{s+1}}=J_{s}+D\left(R_{s}^{\prime}\right) \sigma_{s}\left\langle n_{1}\right\rangle
\end{gathered}
$$

\subsection{Melting Kinetics}

The melting transition in nanoparticles is more complicated than in the bulk; a sizedependent depression of the equilibrium melting point has been observed in free-standing nanoparticles, while for particles embedded in a glass a large hysteresis centered on or near the bulk melting point is observed[14, 18]. For simplicity, the volume change on melting will be ignored; in reality this contributes a stress term to the energy balances. Additional stress terms due to thermal expansion mismatch are also neglected. Particles are assumed to be spherical irrespective of size, as in the absorption and desorption rates. A thermodynamic analysis[14] of an embedded particle gives for the equilibrium melting point depression

$$
\Delta T=\frac{3 T_{m}}{L R}\left(\gamma_{L M}-\gamma_{S M}\right)
$$

with $T_{m}$ the bulk melting point, $L$ the latent heat of melting, and $\gamma_{L M}$ and $\gamma_{S M}$ the liquid-matrix and solid-matrix interface energies[14]. For the case of germanium in silica, no equilibrium melting point depression is observed, so these two surface energies can be taken to be equal and the bulk melting point used. A large hysteresis loop, however, is observed[14]; this can be explained in terms of the kinetics of melting. The most likely kinetic pathway for melting of small particles is nucleation at a surface atom followed by propagation of a spherical cap through the particle. The free energy barrier to melting (freezing) is given by the maximum of

$$
\Delta G=V L \frac{\Delta T}{T_{m}}+A_{S L} \gamma_{S L}
$$

with $V$ the volume of the nucleus, $L$ the volumetric latent heat of melting, $\frac{\Delta T}{T_{m}}$ the overheating $\Delta T=T_{m}-T$ (under-cooling $\Delta T=T-T_{m}$ ), $A_{S L}$ the area of the solid-liquid boundary, and $\gamma_{S L}$ the interface energy. The nucleus volume and boundary areas are those of a spherical lens with a contact angle of $\pi / 2$ because $\gamma_{L M}=\gamma_{S M}$. The maximum in this energy gives a critical nucleus size and a free energy barrier $\Delta G^{*}$ to melting that is temperature- and size-dependent. Using Boltzmann statistics and this energy barrier, 
a melting and freezing transition rate can be calculated for any given particle size and temperature. Assuming a per-atom attempt frequency $\nu$ and that only atoms located within an atomic diameter of the surface may participate in nucleation, the particles of size $j$ at temperature $T$ have a melting transition rate of

$$
\left.\frac{d\left\langle n_{j, l}\right\rangle}{d t}\right|_{\text {melt }}=\left\langle n_{j, s}\right\rangle \nu N_{\text {surf }}(j) \exp \left(\frac{-\Delta G^{*}(j, T)}{k T}\right)
$$

where $\left\langle n_{j, l}\right\rangle$ and $\left\langle n_{j, s}\right\rangle$ are the number densities of liquid and solid particles of size $j$, respectively; $N_{\text {surf }}(j)$ is the number of atoms in a $j$-cluster at the surface and participating in nucleation. The temperature used is that of the relevant particle's edge, since nucleation begins at the surface. The kinetic framework lends itself immediately to the rate equations model; two populations of particles, solid and liquid, interact with each other. Each population experiences absorption and desorption as described above, interacting with a shared field of monomers; the two populations interact by freezing and melting transitions. Nucleation of 2-clusters from the shared monomer field is handled by assigning the cluster to be liquid whenever the 2-cluster melting rate is faster than the 2-cluster freezing rate and solid otherwise; this is equivalent to the temperature being above or below the equilibrium melting point. The full melting and freezing rate equations for liquid clusters $s \geq 2$ are:

$$
\begin{gathered}
\frac{d\left\langle n_{2, l}\right\rangle}{d t}=2 \nu\left(\left\langle n_{2, s}\right\rangle e^{\left(\frac{-\Delta G_{m}^{*}(2, T)}{k T}\right)}-\left\langle n_{2, l}\right\rangle e^{\left(\frac{-\Delta G_{f}^{*}(2, T)}{k T}\right)}\right)+\left\{\begin{array}{cc}
D \sigma_{1}\left\langle n_{1}\right\rangle^{2}, & T>T_{m} \\
0, & T<T_{m}
\end{array}\right. \\
\frac{d\left\langle n_{j, l}\right\rangle}{d t}=\nu N_{\text {surf }}(j)\left(\left\langle n_{j, s}\right\rangle e^{\left(\frac{-\Delta G_{m}^{*}(j, T)}{k T}\right)}-\left\langle n_{j, l}\right\rangle e^{\left(\frac{-\Delta G_{f}^{*}(j, T)}{k T}\right)}\right)
\end{gathered}
$$

with $\Delta G_{m}^{*}$ and $\Delta G_{f}^{*}$ the barrier to melting and freezing respectively. The rate equations for the solid particles are given by changing subscripts appropriately and reversing the conditional for 2-cluster formation. The final term in eq. (30) replaces the first term in eq. (14) for 2-clusters; otherwise the equations for nucleation, growth and coarsening (13 and 14), and melting and freezing (30 and 31) can be combined straightforwardly.

The latent heat of melting must be accounted for in the temperature evolution of the sample, as for the experimental laser power and particle concentration the latent heat for transforming all of the germanium is a significant fraction of the total energy input. The latent heat is tracked by the total mass in each phase, neglecting the size effects on the latent heat but assuming that monomers remain solid for this purpose. The energy that goes into latent heat during each update of the particle distribution is subtracted over the next timestep as a residual power loss to the matrix.

\subsection{Melt Properties}

Several properties including optical and thermal properties change upon melting; however, the most significant effect in this case seems to be the equilibrium solubility outside 
a liquid vs. a solid particle. The solubility $n_{\infty}=n_{0} \exp \left(-E_{f} / k T\right)$ is defined as the number of monomers in equilibrium with a flat surface of a reference state. The formation energy $E_{f}$ is the energy required to remove an atom from the reference state and place it into the matrix; this can be considered a sum[19]

$$
E_{f}=\Delta H_{\text {sub }}+\Delta E_{\text {ion }}-\Delta E_{\text {binding }}-\Delta E_{\text {remainder }}
$$

of the heat of sublimation, the relevant ionization energy (in this case zero, as the Ge atoms in silica should remain neutral), the binding energy of the solute to the matrix, and a remainder energy comprising the image charge heat of formation, the electron affinity, and other effects. The last three terms are independent of the phase of the particle, since a thermodynamic pathway can be constructed in which the atom first sublimates or vaporizes, then ionizes and binds to the matrix. Thus $\Delta E_{f, \text { melting }}=$ $\Delta H_{\text {vap }}-\Delta H_{\text {sub }}=-\Delta H_{\text {melt }}$, the latent heat. This reduction in the formation energy has the effect of drastically increasing the effective solubility of germanium in the matrix, dissolving clusters.

\section{Results and Discussion}

The parameters used to generate these results are literature values for the properties of germanium, silica, and silicon, unless otherwise noted. An important deviation is that in order to decrease the computational expense, a $\mathrm{Ge}-\mathrm{SiO}_{2}$ interface energy of 0.2 $\mathrm{J} / \mathrm{m}^{2}$ is used as a default value to calculate the absorption and desorption rate constants instead of a more realistic $0.7[14]$ to $1.5[5] \mathrm{J} / \mathrm{m}^{2}$. This modification has the major effect of reducing the critical cluster size of nucleation[5], allowing particles to form at lower overall system sizes and decreasing computation time.

The laser schedule used, mimicking typical experiments, is a 30-ns pulse at $248 \mathrm{~nm}$ wavelength, with a total fluence of $0.3 \mathrm{~J} / \mathrm{cm}^{2}$. The laser power is assumed to be constant over the pulse time. The laser power is set to zero after $30 \mathrm{~ns}$ simulation time and the system is allowed to cool. The initial temperature of the system and surroundings is set to $300 \mathrm{~K}$. Starting particle size distributions are created by an implant simulation conducted at a constant $300 \mathrm{~K}$.

The simulation under these conditions shows that the nanoparticles reach a temperature exceeding $2800 \mathrm{~K}$ due to the large laser power and the low thermal conductivity of the silica matrix. The temperatures of the particles vary by size and phase, with large particles retaining more heat than small particles; the temperatures differences between large particles, small particles, and the matrix in the implanted layer is a few degrees K. The majority of the particles are molten after roughly $8 \mathrm{~ns}$, corresponding to a melt temperature of $1398 \mathrm{~K}$; this is substantially above the bulk melting temperature of $1210.6 \mathrm{~K}$ due to the kinetics of melting embedded particles. The majority of the particles are solid again $27 \mathrm{~ns}$ after the end of the laser pulse, corresponding to a temperature of $1247 \mathrm{~K}$; we postulate that this is due to the high desorption rate of liquid particles 


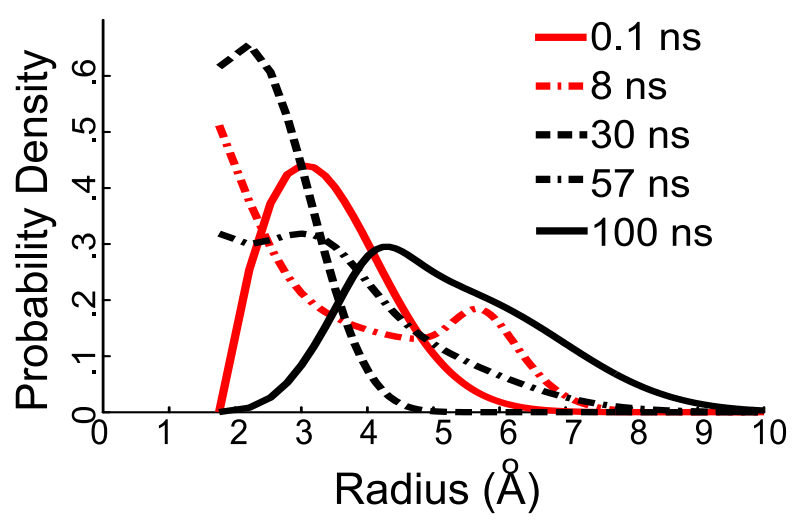

Figure 1: Evolution of the nanoparticle size distribution over time. Size distributions are presented at the beginning of the pulse $(0.1 \mathrm{~ns})$, during melting $(8 \mathrm{~ns})$, at the end of the pulse (30 ns), during freezing (57 ns), and after cooling (100 ns). The cutoff in size at $1.7 \AA$ radius is single atoms.

favoring growth of the solid particles rather than melting and freezing kinetics alone. In fact, suppressing absorption and desorption in the simulation, i.e. allowing no change in the distribution, shows the particles freezing below the bulk melting temperature, as expected from the kinetics. Slight plateaus in the temperature profile are observed at melting and freezing, corresponding to the latent heat of the transformation.

Although the peak temperature in the implanted layer is very high, heat is dissipated very quickly, and the silicon substrate remains relatively cool. Just $100 \mathrm{~ns}$ after the laser pulse ends, the temperature of the implanted layer has dropped to $589 \mathrm{~K}$, corresponding with experimental evidence of a fast quench rate[3]. Because of the relatively high thermal conductivity of silicon, the highest temperature the substrate reaches is 458 $\mathrm{K}$ at the surface. It is worth noting that the fast quench is not due to the matrix remaining at a lower temperature than the particles during the pulse, but rather due to the unimplanted silica on either side of the implanted layer remaining relatively cool.

In addition to the temperatures reached, the resulting particle size and size distribution are of interest; the particle sizes affect subsequent properties. Size distributions are here presented in radius space (rather than atoms per cluster) as probability densities, i.e. the probability that a randomly chosen particle will have a given radius. At high temperatures, two major effects change the distribution: faster diffusion leads to coarsening, tending to increase the average size, while higher temperatures increase the solubility, tending to increase the number of dissolved atoms and decrease the average size. The distribution during melting in figure 1 shows this interplay, as the initial singlepeaked distribution splits into a high single-atom peak at $1.7 \AA$ radius and a lower peak at larger sizes. Well above the melting point, dissolution dominates, giving a single peak at one- and two-atom clusters. As the temperature comes down through solidification, the distribution bifurcates again (fig. 1) as small clusters start to grow while a large 
number of atoms remain dissolved; as the temperature continues to drop the clusters coarsen while retaining the bimodal distribution shape.

\subsection{Absorption bounds}

Comparing the upper- and lower-bound assumptions for the laser absorption, the results are qualitatively very similar, but the peak temperature reached using the lower bound is just $78 \%$ of the higher bound (fig. 2a). The thermal budget is smaller, so there is less change in the particle size distribution, and the final average particle size is smaller (fig. 2b-d). The time spent above the melting temperature is shorter and the peak temperature is lower, so fewer clusters dissolve completely, explaining the larger particle size (fig. 2b) during the laser pulse and until solidification. The upper bound is used in the remainder of the results.

\subsection{Annealing}

Instead of starting the PLM simulation from an as-implanted distribution, an intermediate rapid thermal anneal (RTA) can be added. To accomplish this, the simulation is run with zero laser intensity, instead changing the base temperature over time. To mimic experimental conditions, the base temperature schedule ramps linearly to the target anneal temperature (in this case $300^{\circ} \mathrm{C}$ ) over the course of two seconds, holds for six seconds, and ramps back down. This has the effect of increasing the average particle size and changing the distribution shape from positive to negative skewness (fig. 3). However, the resulting particle distribution after PLM is nearly identical; the PLM final distribution seems to be independent of the input distribution. In fact, the RTA/PLM cycle can be repeated several times, with each PLM step giving identical results(fig. 4b), and each RTA step except the first taking an identical initial distribution and thus returning an identical final distribution. This result corresponds with recent experiments[3], which have shown that the process is repeatable in an AuGe alloy nanoparticle system with nanoparticles still present after ten PLM/RTA cycles, and suggests that no significant degradation in the size distribution will occur. It should be noted, however, that this result may be an artifact of the small interface energy; the smaller particle size means the laser is able to "reset" the distribution to a single peak of very small particles, while in a system with higher interface energy, some very large particles may survive the pulse and lead to a different distribution.

The final distribution in this case is characteristic of only the laser fluence. A temperature (roughly $2300 \mathrm{~K}$ ) is reached at which both the desorption rate and the diffusivity are high enough that the system reaches its equilibrium within a nanosecond. At the peak temperature of $2800 \mathrm{~K}$, the desorption rates are such that a 50-atom liquid particle desorbs well over 50 atoms in $0.1 \mathrm{~ns}$; the diffusion length in the same $0.1 \mathrm{~ns}$ is $2.8 \mathrm{~nm}$, while the particle density is just over one particle per $\mathrm{nm}^{3}$. The melt rate for the same 50 -atom particle is $4.5 \times 10^{12} \mathrm{~s}^{-1}$, while the freezing rate is negligible. The combined 

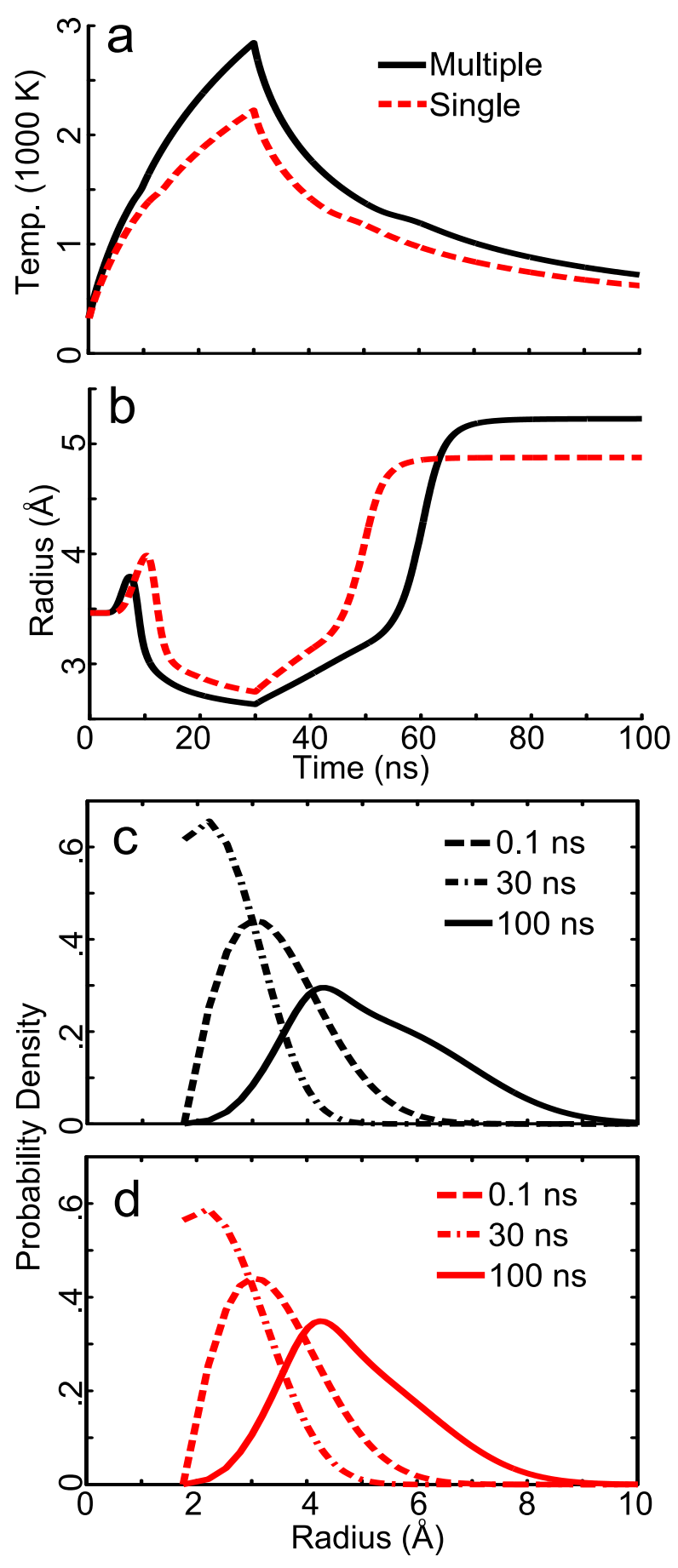

Figure 2: Comparison of the multiple-reflections upper bound on the laser absorption and single-pass lower bound. Temperature profiles (a) and average radii (b) over time for both simulations. Size distributions of the initial state, at the end of the laser pulse, and after cooling for $70 \mathrm{~ns}$ for multiple absorptions (c) and single-pass (d) case. 


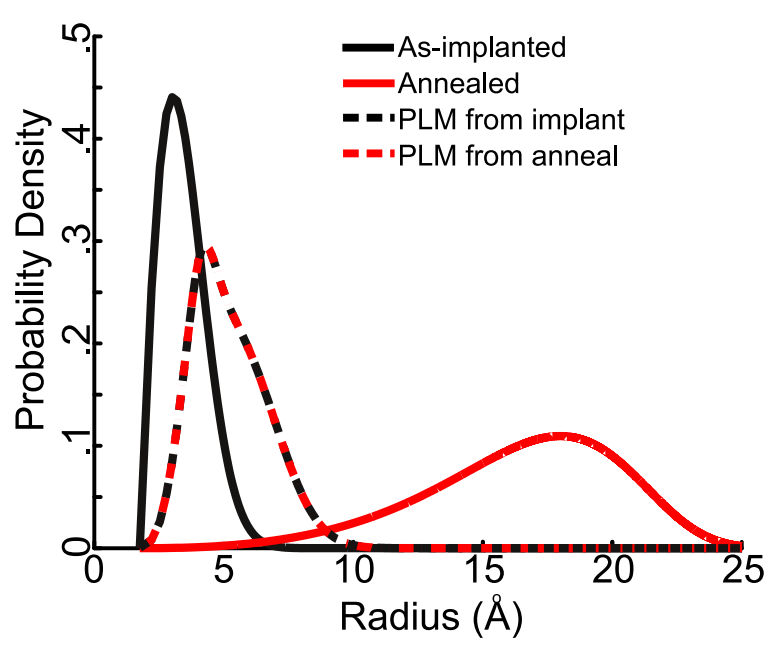

Figure 3: Distributions before and after PLM, starting from the as-implanted and 6second RTA final distributions. The distributions after PLM overlay each other.

effect of these rates is to quickly bring any starting distribution to a high-temperature equilibrium dominated by one-, two- and three-atom clusters in a liquid state. The final distribution then is simply a result of the cooling rate, which determines the thermal budget for coarsening.

\subsection{Laser power}

Similar to reducing the laser absorption, reducing the laser power provided gives a lower temperature profile, a smaller final particle size, and less change between the initial and final distributions (fig. 5c). As long as the particles reach a temperature well above the melting point, the behavior is qualitatively the same. If the particles do not melt at all, as at $0.1 \mathrm{~J} / \mathrm{cm}^{2}$ laser fluence, then PLM functions like a very short anneal (fig. 5e), increasing the average particle size slightly. A small amount of size distribution narrowing is seen as the particles grow.

An intermediate laser fluence of $0.15 \mathrm{~J} / \mathrm{cm}^{2}$ causes the particles to reach the melting point, but due to kinetics not all of the particles melt; a small number of solid particles survive and coarsen (fig. 5d). This results in a strongly bimodal distribution after PLM, with a large peak below $5 \AA$ radius and a smaller peak around $13 \AA$. This kind of bimodal distribution provides the possibility of narrowing the size distribution as the large number of small particles serve as a feedstock for the larger particles to grow without cannibalizing each other as in Ostwald ripening. To explore this possibility, a one-second RTA simulation was run starting from this output distribution; the results are shown in figure 6 . This distribution is substantially narrowed relative to the average size, with a distribution FWHM of $14.9 \%$ of the average radius. The length of the RTA step was chosen to give the sharpest peak, when most of the mass has transferred from 


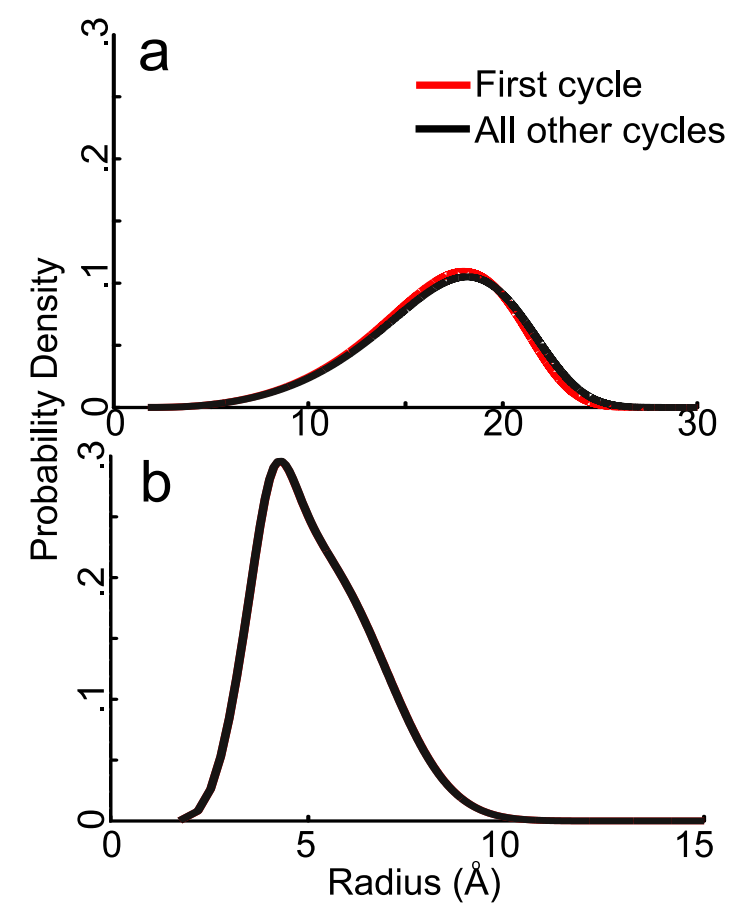

Figure 4: Final particle size distributions during a repeated RTA (a) and PLM (b) cycle. Results from all but the first RTA step overlay each other. 

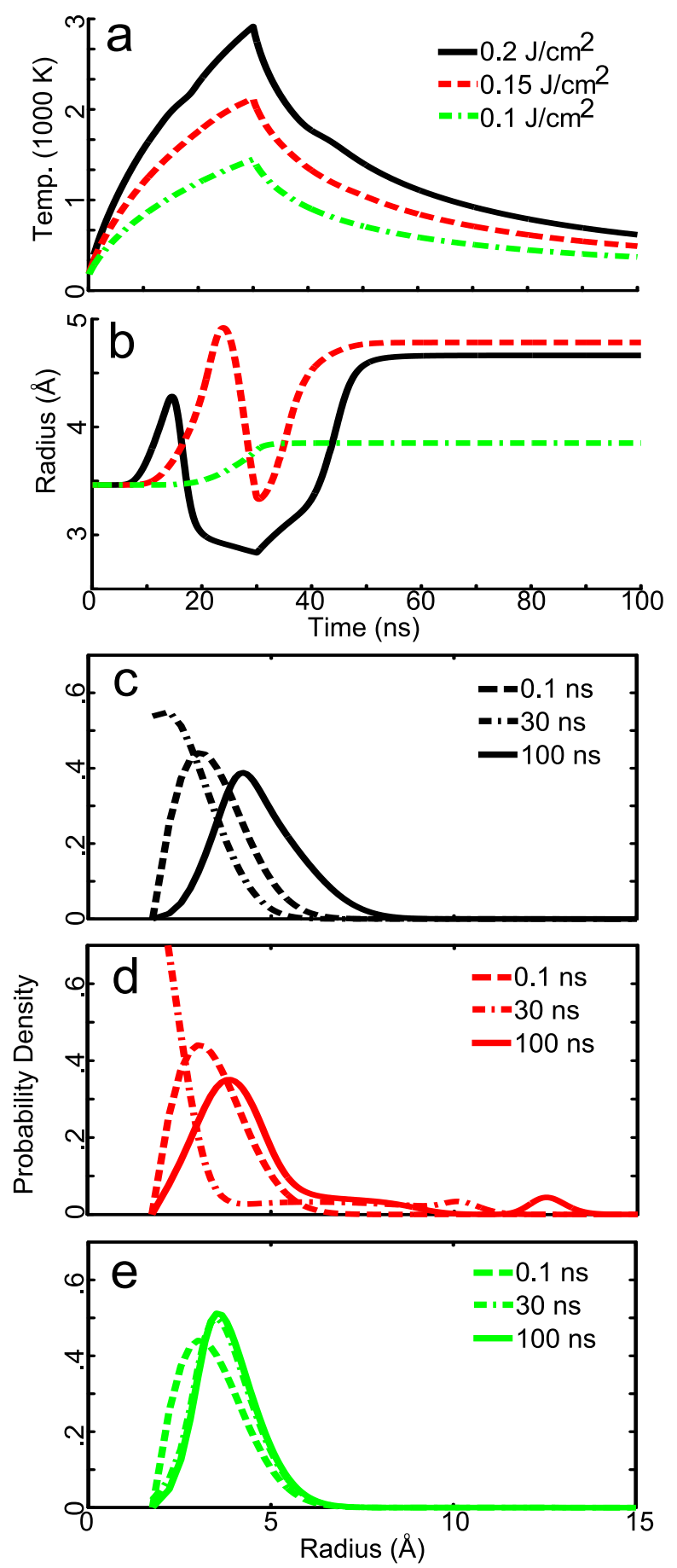

Figure 5: Comparison of lower laser powers. Pulse time is constant and total laser fluence is decreased from $0.2 \mathrm{~J} / \mathrm{cm}^{2}$ (black, c) to $0.15 \mathrm{~J} / \mathrm{cm}^{2}$ (red, d) to $0.1 \mathrm{~J} / \mathrm{cm}^{2}$ (green, e). Temperature profiles (a) and average radii (b) are shown for each fluence. 


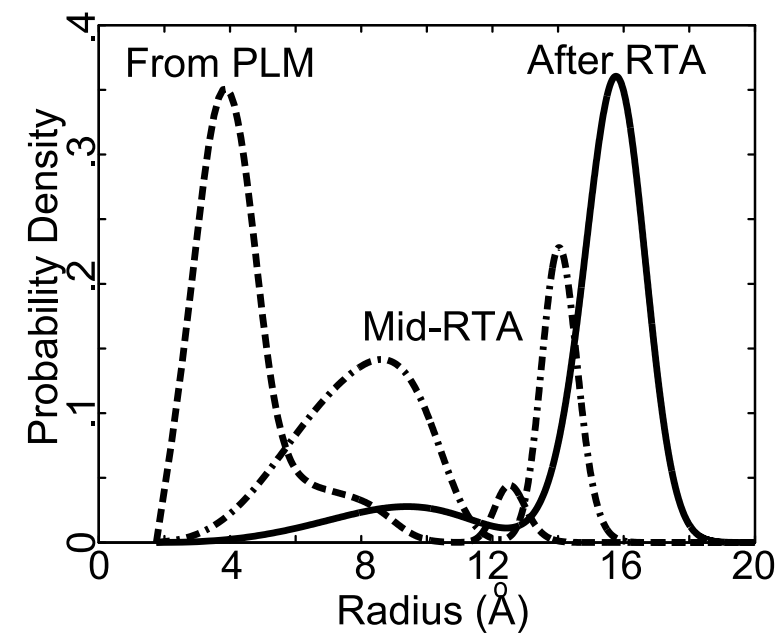

Figure 6: Size-focusing using fine-tuned PLM and RTA; final distribution (green) has a FWHM just $14.9 \%$ of the average radius.

the small-particle peak to the large-particle peak but Ostwald ripening of the larger particles has not yet had a large broadening effect.

The size distribution splitting predicted here is analogous to previous work on coarsening during heteroepitaxial island formation[20]. In that case, a shape transition as a function of size caused a discontinuity in the chemical potential, which in turn caused the transformed particles to grow quickly at the expense of the smaller particles. A narrow size distribution resulted. In this case, the chemical potential difference is reached in two ways: first, partial melting creates two populations of particles whose sizes diverge. Second, even after all particles have returned to the solid state, a discontinuity exists because of the bimodal distribution itself. While the bimodal distribution persists, Ostwald ripening consumes the small-particle peak without broadening the large-particle peak, leading to a sharp overall distribution. A characteristic size can be identified for this process as the largest particles to melt; particles smaller than this will shrink into the small-particle peak, while larger particles will grow during cooling and form the large-particle peak. For this simulation, the particle size for which half the particles were liquid and half solid at the peak temperature is 174 atoms, or $9.8 \AA$ Aradius.

\subsection{Interface Energy}

Although computationally expensive, the model does allow larger particle-matrix interface energies; simulations at an interface energy of $0.5 \mathrm{~J} / \mathrm{m}^{2}$ are shown in figure 7 . Realistic interface energies are closer to $0.7[14]$ to $1.5[5] \mathrm{J} / \mathrm{m}^{2}$. The major effect of increasing the interface energy in the implantation simulations is to increase the average sizes via the critical cluster size, leading to a simple scaling law[5]; however, in the PLM case the behavior is more complex. Although the temperature profile for a given laser 
fluence is nearly identical for different interface energies, the particle distribution continues to evolve for a longer time, necessitating a longer simulation (fig. 7b). The higher interface energy discourages the formation of subcritical 2-atom clusters, leaving more dissolved single atoms in the matrix over time. For a fluence of $0.3 \mathrm{~J} / \mathrm{cm}^{2}$, the final size distribution has changed qualitatively; the main peak at $5 \AA$ radius is fairly broad, and there is a small peak of frozen-in monomers (fig. 7c). Additionally, there is a low, broad peak at roughly $27 \AA$ radius, not shown. This suggests that at higher, more realistic interface energies the final distribution becomes more bimodal or multimodal, agreeing with experimental evidence that a Ge-Sn nanoparticle system under PLM results in large particles surrounded by a "halo" of smaller material[1].

However, at the lower fluences that show bifurcation in the small interface energy case, e.g. $0.15 \mathrm{~J} / \mathrm{cm}^{2}$, the behavior is qualitatively the same: a bimodal distribution is seen in figure $7 \mathrm{~d}$ similar to that in $5 \mathrm{~d}$, although the relative heights of the peaks are switched. Both have a shouldered peak at small sizes and a sharp peak at large sizes. This distribution, without a following RTA step, has a FWHM just $16 \%$ of the average radius, significant narrowing over the as-implanted $64 \%$. This suggests that the possibility of narrowing distributions under PLM exists at realistic interface energies.

\section{Conclusion}

A model of pulsed laser melting has been created which tracks the particle size distribution and various temperatures within the sample. The model includes nucleation and growth, dissolution, and melting of the nanoparticles, as well as absorption of the laser and dissipation of heat through the system. Ion beam synthesis and rapid thermal annealing are also included. The results provide for comparison between possible laser powers to fine-tune processing and correspond with experimental results regarding cycling RTA and PLM steps for possible phase change material applications. In particular, simulations modeling experimental conditions show no change in the size distribution over multiple RTA/PLM cycles. Additionally, a route for narrowing the size distribution of embedded particles is suggested; reducing the laser power should give a bifurcated distribution which can be further annealed to produce a very narrow (15\% width) size distribution in embedded particles.

\section{Acknowledgements}

This work was supported by the Director, Office of Science, Office of Basic Energy Sciences, Materials Sciences and Engineering Division, of the U.S. Department of Energy under Contract No. DE-AC02-05CH11231. 

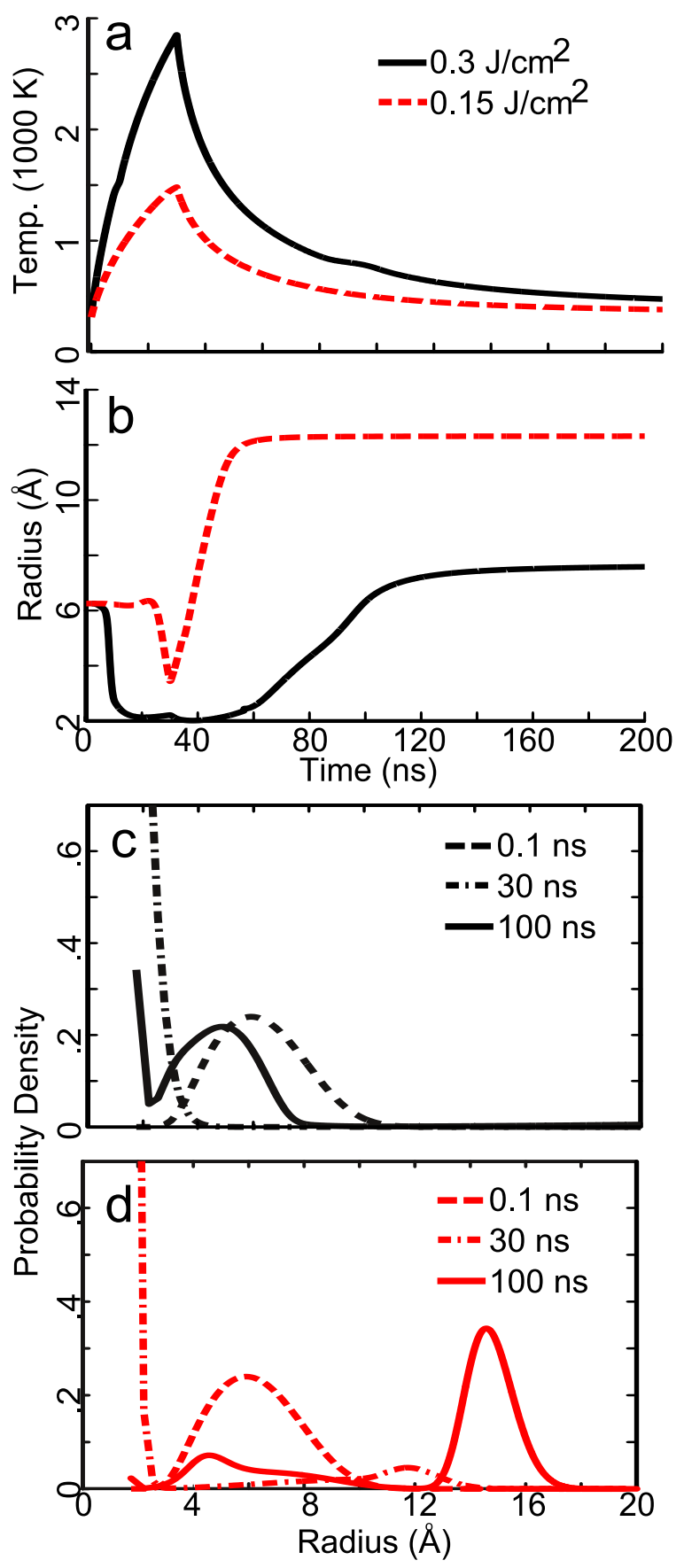

Figure 7: Selected simulations at a interface energy $0.5 \mathrm{~J} / \mathrm{m}^{2}$. PLM at a laser fluence of $0.3 \mathrm{~J} / \mathrm{cm}^{2}$ (black, c) and $0.15 \mathrm{~J} / \mathrm{cm}^{2}$ (red, d) are shown. Temperature profiles (a) and average radii (b) are shown for each fluence. Compare figures 2 and 5. 


\section{References}

[1] S. J. Shin, J. Guzman, C.-W. Yuan, C. Y. Liao, C. N. Boswell-Koller, P. R. Stone, O. D. Dubon, A. M. Minor, M. Watanabe, J. W. Beeman, K. M. Yu, J. W. Ager III, D. C. Chrzan, and E. E. Haller. Nano Lett., 10:2794, 2010.

[2] S. J. Shin, J. Guzman, C.-W. Yuan, C. Y. Liao, P. R. Stone, O. D. Dubon, A. M. Minor, M. Watanabe, K. M. Yu, J. W. Ager III, D. C. Chrzan, and E. E. Haller. Mater. Res. Soc. Symp. Proc., 1184:HH04-08, 2009.

[3] J. Guzman, C. N. Boswell-Koller, J. W. Beeman, K. C. Bustillo, T. Conry, O. D. Dubon, W. L. Hansen, A. X. Levander, C. Y. Liao, R. R. Lieten, C. A. Sawyer, M. P. Sherburne, S. J. Shin, P. R. Stone, M. Watanabe, K. M. Yu, J. W. Ager III, D. C. Chrzan, and E. E. Haller. Appl. Phys. Lett., 98:193101, 2011.

[4] E. M. Chan, C. Xu, A. W. Mao, G. Han, J. S. Owen, B. E. Cohen, and D. J. Milliron. Nano Lett., 10:1874, 2010.

[5] C. W. Yuan. Modeling Size Distribution Evolution of Ion-Beam-Synthesized Nanoclusters in Amorphous Silica. PhD thesis, University of California, Berkeley, 2009.

[6] C.-W. Yuan, D. O. Yi, I. D. Sharp, S. J. Shin, C. Y. Liao, J. Guzman, J. W. Ager III, E. E. Haller, and D. C. Chrzan. Phys. Rev. B, 80:134121, 2009.

[7] T. Kim, M. R. Pillai, M. J. Aziz, M. A. Scarpulla, O. D. Dubon, K. M. Yu, J. W. Beeman, and M. C. Ridgway. J. Appl. Phys., 108:013508, 2010.

[8] R. F. Wood and G. E. Giles. Phys. Rev. B, 23:2923, 1981.

[9] J. Trice, D. Thomas, C. Favazza, R. Sureshkumar, and R. Kalyanaraman. Phys. Rev. B, 75:235439, 2007.

[10] J. Trice, C. Favazza, D. Thomas, H. Garcia, R. Kalyanaraman, and R. Sureshkumar. Phys. Rev. Lett., 101:017802, 2008.

[11] C.-W. Yuan, D. O. Yi, I. D. Sharp, S. J. Shin, C. Y. Liao, J. Guzman, J. W. Ager III, E. E. Haller, and D. C. Chrzan. Phys. Rev. Lett., 102:146101, 2009.

[12] D. O. Yi. Modeling nucleation, growth and stress: Ge nanocrystals embedded in $\mathrm{SiO}_{2}$. PhD thesis, University of California, Berkeley, 2005.

[13] D. O. Yi, M. H. Jhon, I. D. Sharp, Q. Xu, C. W. Yuan, C. Y. Liao, J. W. Ager III, E. E. Haller, and D. C. Chrzan. Phys. Rev. B, 78:245415, 2008.

[14] Q. Xu, I.D. Sharp, C.W. Yuan, D.O. Yi, C.Y. Liao, A.M. Glaeser, A.M. Minor, J.W. Beeman, M.C. Ridgway, P. Kluth, J.W. Ager III, D.C. Chrzan, and E.E. Haller. Phys. Rev. Lett., 97:155701, 2006. 
[15] L. Viña, S. Logothetidis, and M. Cardona. Phys. Rev. B, 30:1979, 1984.

[16] G.E. Jellison Jr., D.H. Lowndes, D.N. Mashburn, and R.F. Wood. Phys. Rev. B, 34:2407, 1986.

[17] W. Andrä, C.G. d'Ambly, R. Hergt, I. Hilger, and W.A. Kaiser. J. Magn. Magn. Mat., 194:197, 1999.

[18] H.H. Anderson and E. Johnson. Nucl. Instr. Meth. Phys. Res. B, 106:480, 1995.

[19] J.D. McBrayer, R.M. Swanson, and T.W. Sigmon. J. Electrochem. Soc.: Solid State Science and Technology, 133:1242, 1986.

[20] F. M. Ross, J. Tersoff, and R. M. Tromp. Phys. Rev. Lett., 80:984, 1998. 


\section{DISCLAIMER}

This document was prepared as an account of work sponsored by the United States Government. While this document is believed to contain correct information, neither the United States Government nor any agency thereof, nor The Regents of the University of California, nor any of their employees, makes any warranty, express or implied, or assumes any legal responsibility for the accuracy, completeness, or usefulness of any information, apparatus, product, or process disclosed, or represents that its use would not infringe privately owned rights. Reference herein to any specific commercial product, process, or service by its trade name, trademark, manufacturer, or otherwise, does not necessarily constitute or imply its endorsement, recommendation, or favoring by the United States Government or any agency thereof, or The Regents of the University of California. The views and opinions of authors expressed herein do not necessarily state or reflect those of the United States Government or any agency thereof or The Regents of the University of California. 